\title{
The Effectiveness of Rehabilitation Program of Dohsa-Hou on Perceived Stress, Meta-Worry and Quality of Life of Hemodialysis Patients
}

\author{
Azita Behjat ${ }^{1}$ (D), Zahra Mirshamsi ${ }^{2}$ (D), Shahla Karbalaei Saleh ${ }^{3}$ (D), Zahra DashtBozorgi ${ }^{4, *}$ (D) \\ ${ }^{1}$ MA of Clinical Psychology, Department of Psychology, Yazd Branch, Islamic Azad University, Yazd, Iran \\ 2 PhD Student of Psychology, Department of Psychology, Faculty of Humanities Sciences, University of Science and Arts, Yazd, \\ Iran \\ ${ }^{3}$ MA of General Psychology, Department of Psychology, Ramhormoz Branch, Islamic Azad University, Ramhormoz, Iran \\ ${ }^{4}$ Assistant Professor, Department of Psychology, Ahvaz Branch, Islamic Azad University, Ahvaz, Iran \\ * Corresponding author: Zahra DashtBozorgi, Assistant Professor, Department of Psychology, Ahvaz Branch, Islamic Azad \\ University, Ahvaz, Iran. E-mail: zahradb2000@gmail.com \\ How to Cite this Article: \\ Behjat A, Mirshamsi Z, Karbalaei Saleh Sh, DashtBozorgi Z. The Effectiveness of Rehabilitation Program of Dohsa-Hou on \\ Perceived Stress, Meta-Worry and Quality of Life of Hemodialysis Patients. Iran J Rehabil Res Nurs. 2020;7(1):44-54. \\ DOI: 10.29252/ijrn.7.1.44
}

Received: 08 Feb 2020

Accepted: 03 Jun 2020

Keywords:

Health

Hemodialysis

Meta-Worry

Quality of Life

Rehabilitation

Stress

(C) 2020 Iranian Journal of

Rehabilitation Research in Nursing

\begin{abstract}
Introduction: Hemodialysis patients face difficulties and limitations in physical, social and psychological functioning which from their psychological problems cited to increase perceived stress and meta-sorry and decrease quality of life. Regarded to the effectiveness of rehabilitation program of Dohsa-hou on many psychological characteristics, present research aimed to determine the effect of rehabilitation program of Dohsa-hou on perceived stress, meta-worry and quality of life in hemodialysis patients.

Methods: The method of present research was quasi-experimental with pretest and posttest design with control group. The research population was patients referred to hemodialysis ward of Shahid Mofatah Hospital of Varamin city in 2018 year. The research sample was 34 patients with low quality of life which were selected by purposeful sampling method and randomly replaced into two equal groups. The experimental group trained 11 sessions of 45 minutes with rehabilitation program of Dohsa-hou and the control group was replaced on the waiting list for training. Data collected with specifications demographic form, perceived stress questionnaire, subscale of meta-worry in anxious thought scale and revised health related quality of life questionnaire and analyzed with using methods of Chi-square, t-test and multivariate analysis of covariance.

Results: The findings showed that between the experimental and control groups there was no significant difference in terms of gender, age, education and marriage $(P>0.05)$. Also, between groups in the pretest stage there was no significant difference in terms of perceived stress, metaworry and health related quality of life $(P>0.05)$, but in the posttest stage there was significant difference in terms of all three variables $(\mathrm{P}<0.05)$. In the other words, rehabilitation program of Dohsa-hou led to decreased perceived stress and meta-worry and significantly increased health related quality of life in hemodialysis patients $(P<0.05)$.

Conclusions: Based on the results, it is recommended that therapists and clinical psychologists along with other approaches and therapies, to use from the rehabilitation program of Dohsahou to improve the characteristics related health including decrease stress and meta-worry and increase health related quality of life of hemodialysis patients.
\end{abstract}

\section{Extended Abstract}

\section{OBJECTIVE}

$\mathrm{T}$

oday hemodialysis is one of the most common treatment methods of kidney replacement in patients with chronic kidney failure [2]. Chronic kidney disease is a destructive and irreversible process that led to reduces glomerular filtration, reduces the ability of the kidneys to maintain fluids and electrolytes and disrupts the balance of water and urea (uremia) [1]. Hemodialysis patients face difficulties and limitations in physical, social and psychological functioning which from their psychological problems cited to increase perceived stress [6] and meta-sorry [7] and decrease quality of life [2]. Perceived stress is the

Copyright (C) 2020 The Author(s); Published by Iranian Journal of Rehabilitation Research in Nursing. This is an open access article, distributed under the terms of the Creative Commons Attribution-NonCommercial 4.0 International License (http://creativecommons.org/licenses/by-nc/4.0/) which permits others to copy and redistribute material just in noncommercial usages, provided the original work is properly cited. 
amount of stress that a people perceive about situations and events and this stress has a negative impact on their health, well-being and quality of life [8]. Also, the metaworry is transforming normal worry through negative beliefs and evaluations into abnormal worry that plays an important role in reducing health [11]. In addition, the quality of life especially in chronic diseases such as kidney failure is known as a one of the criteria considered in the medical world [14]. Quality of life is a comprehensive and complex concept of individuals' mental evaluation about their sense of welfare and ability to perform physical, psychological and social functions [15]. One of the therapeutic methods to improve many psychological problems is the use of rehabilitation program of Dohsa-hou [17]. This rehabilitation program is one of the complementary therapies methods that balance the people's body awareness and with relax their muscles and brain increasing their awareness of themselves and others and the therapist activates both the client's mind and it also strengthen the cooperation and human communication [15]. Therefore, present research aimed to determine the effect of rehabilitation program of Dohsa-hou on perceived stress, meta-worry and quality of life in hemodialysis patients.

\section{MATERIALS AND METHODS}

The method of present research was quasi-experimental with pretest and posttest design with an experimental and control groups. The population of this research was consisted of patients referred to hemodialysis ward of Shahid Mofatah Hospital of Varamin city in 2018 year with number of 573 people. From the between of population members were selected 34 individuals with low quality of life after reviewing the inclusion criteria to study with purposeful sampling method and randomly were replaced into two equal groups including experimental and control groups (each group 17 people). The experimental group trained individually 11 sessions of 45 minutes (two sessions per week) with rehabilitation program of Dohsa-hou by a clinical psychologist with degree of rehabilitation program of Dohsa-hou in one of the psychological services clinics of Varamin city and the control group was replaced on the waiting list for training. After expressing ethical considerations (principles of secrecy, confidentiality of personal information, privacy of the subjects and etc.) and receive from of consent conscious participation in research the research firm to collect data use from specifications demographic form, perceived stress questionnaire, subscale of meta-worry in anxious thought scale and revised health related quality of life questionnaire. In the present study the reliability were calculated by Cronbach's alpha method for perceived stress, meta-worry and health related quality of life in hemodialysis patients $0.85,0.79$ and 0.90 , respectively. Data were analyzed with using methods of Chi-square, t-test and multivariate analysis of covariance in SPSS software version 24 in significant level of $\mathrm{P}<0.05$.

\section{RESULTS}

The findings showed that between the experimental and control groups there was no significant difference in terms of gender, age, education and marriage $(\mathrm{P}>0.05)$. Also, between the experimental and control groups in the pretest stage there was no significant difference in terms of perceived stress, meta-worry and health related quality of life $(P>0.05)$, but between the mentioned groups in the posttest stage there was significant difference in terms of all three variables of perceived stress, meta-worry and health related quality of life $(\mathrm{P}<$ 0.05) (Table 1). In the other words, rehabilitation program of Dohsa-hou led to significantly decreased perceived stress and meta-worry and significantly increased health related quality of life in hemodialysis patients $(\mathrm{P}<0.05)($ Table 2$)$.

Table 1. T-Test Results

\begin{tabular}{lccc}
\hline Variables & Experimental Group M \pm SD & Control Group M \pm SD & P-Value \\
\hline Perceived stress & & & $>0.05$ \\
Pre-test & $24.76 \pm 4.39$ & $23.15 \pm 4.48$ & $<0.001$ \\
Post-test & $17.25 \pm 2.82$ & $24.01 \pm 4.33$ & $>0.05$ \\
Meta-worry & $16.94 \pm 3.70$ & $16.56 \pm 3.57$ & $<0.001$ \\
$\quad$ Pre-test & $11.41 \pm 2.26$ & $16.72 \pm 3.66$ & $>0.05$ \\
Post-test & & & $<0.001$ \\
Quality of life & $20.36 \pm 1.95$ & $19.51 \pm 3.57$ & \\
Pre-test & $31.24 \pm 2.76$ & $18.74 \pm 1.88$ & \\
Post-test & & & \\
\hline
\end{tabular}

Data in table are presented as Mean \pm SD.

Table 2. MANCOVA Results

\begin{tabular}{lcccccccc}
\hline Variables & Source & Sum of Squares & df & Mean Squares & F & P-Value & Eta Squared & Test Power \\
\hline Perceived stress & Group & 256.39 & 1 & 256.39 & 79.81 & $<0.001$ & 0.79 & 0.89 \\
Meta-worry & Group & 148.64 & 1 & 148.64 & 76.94 & $<0.001$ & 0.77 & 0.88 \\
Quality of life & Group & 235.17 & 1 & 235.17 & 87.22 & $<0.001$ & 0.85 & 0.93 \\
\hline
\end{tabular}

\section{CONCLUSION}

The results indicated the effect of rehabilitation program of Dohsa-hou on the significant decrease of perceived stress and meta-worry and significant increase of health related quality of life in hemodialysis patients. Based on the relatively high statistics of hemodialysis patients and their psychological problems and regarded to this rehabilitation program is a relatively cheap, 
efficient and practical method, it can be used to improve psychological characteristics. As a result, it is recommended that therapists and clinical psychologists along with other approaches and therapies, to use from the rehabilitation program of Dohsa-hou to decrease perceived stress and meta-worry and increase health related quality of life, especially in patients with chronic disorders including hemodialysis patients.

\section{Ethical Considerations}

In this study, ethical points according to the ethical protocols of Helsinki studies were done and received ethical code from Islamic Azad University of Ahvaz branch with number of IR.IAUAhvaz.REC.1397.150. Also, the research consent form was signed by all subjects

\section{Funding of Supports}

This research was conducted at the authors' expense.

\section{Author's Contribution}

Ladies Azita Behjat and Shahla Karbalaei Saleh: Intervention coordination, data collection and data entry to computer, Zahra Mirshamsi: Background collection and writing initial version of article and Zahra DashtBozorgi: intervention, data analysis and writing final version of article, submission, follow-up and editing.

\section{Conflict of Interest}

Regarded to authors declare there was no conflict of interest between them.

\section{Applicable Remarks}

Regarded the high prevalence of kidney disease and the psychological problems of hemodialysis patients including high stress and anxiety and low quality of life, the rehabilitation program of Dohsa-hou could improve the psychological traits, especially reducing perceived stress and meta-worry and enhancing health related quality of life. Therefore, therapists and health care providers can use the results of this research as a new perspective to enhance health indicators and even preventing them in hemodialysis patients. As a result, the use of the rehabilitation program of Dohsa-hou in health and care policies related to chronic diseases such as hemodialysis can play an important role in improving their psychological traits.

\section{Acknowledgments}

The authors hereby express their gratitude and appreciation from research participant and authorities of Shahid Mofatah Hospital of Varamin city. 


\title{
اثربخشى بر نامه توانبخشى دوسا-هو بر استرس ادراكشده، فرانكرانى و كيفيت زنفكى بيماران همودياليزى
}

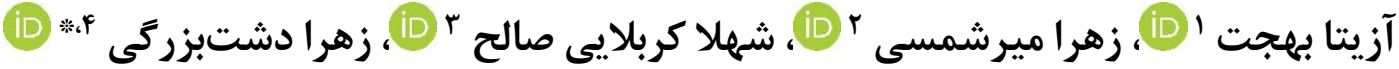

' كارشناس ارشد روانشناسى بالينى، گروه روانشناسى، واحد يزد، دانشحاه آزاد اسلامى، يزد، ايران

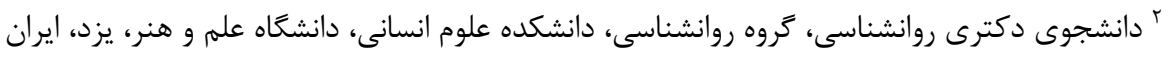

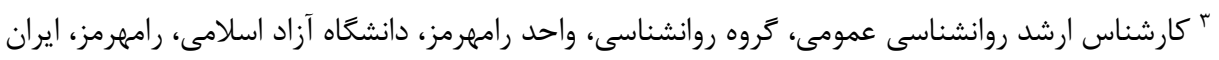

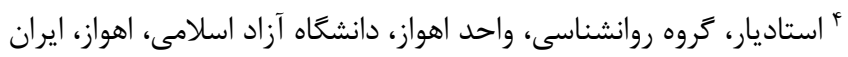

* نويسنده مسئول: زهرا دشتبز گىى، استاديار، گروه روانشناسى، واحد اهواز، دانشكاه آزاد اسلامى، اهواز، ايران. ايميل: zahradb2000@yahoo.com

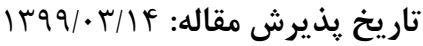
تاريخ دريافت مقاله: 9 (1/1/1/1

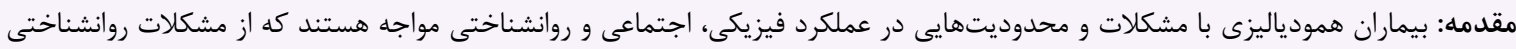

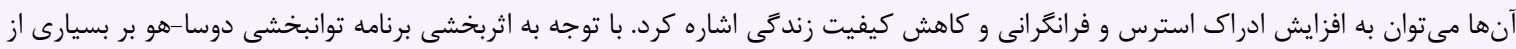

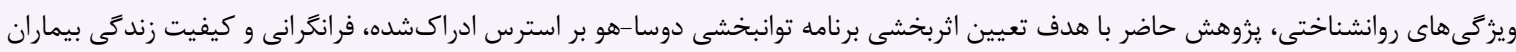

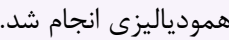

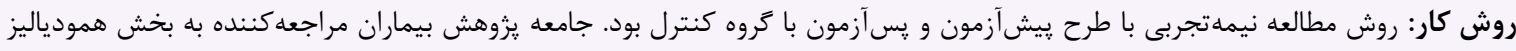

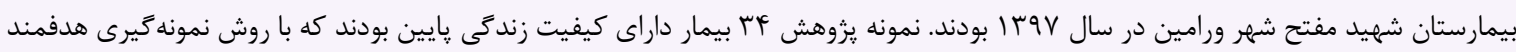

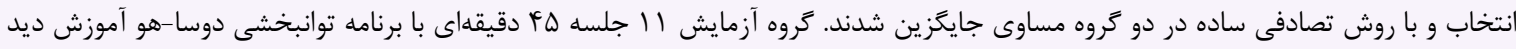

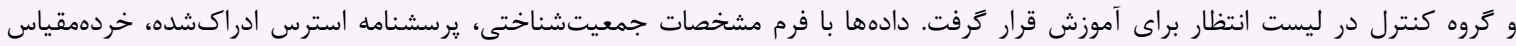

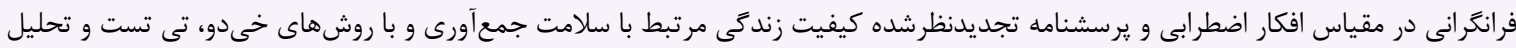
كوواريانس جندمتغيرى تحليل شدند.

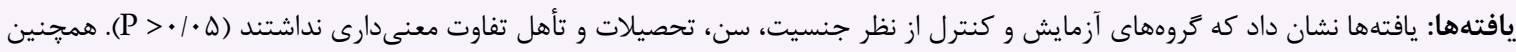

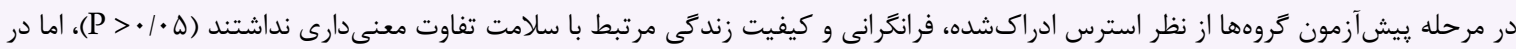

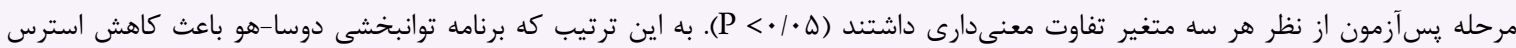

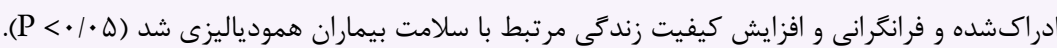

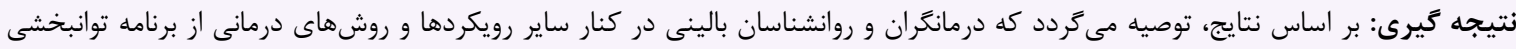

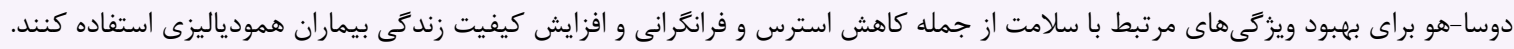

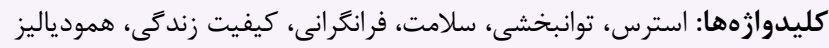

تمامى حقوق نشر براى انجمن علمى يرستارى ايران محفوظ است.

مقدمه

الكتروليتها و برهمزدن تعادل آب و اوره (اورمى) مىشود [1]. اين

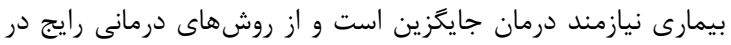

بيمارى مزمن كليه فرايند تخريبى و بركشتنايذيرى است كه سبب

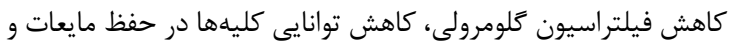


يكى از روشهاى درمانى براى بجبود بسيارى از مشكلات روانشناختى إنى

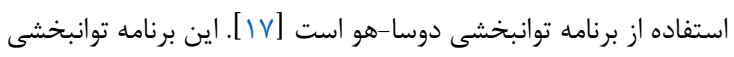

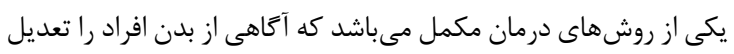

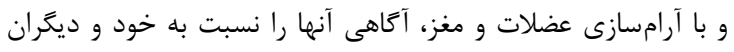

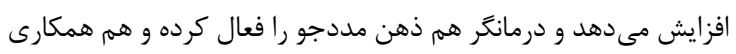

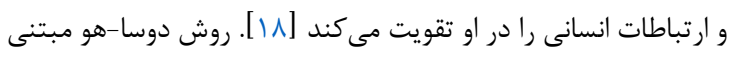

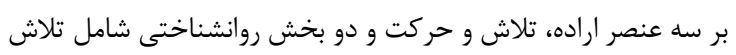

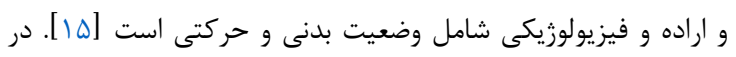

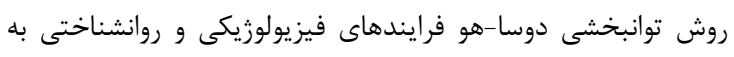

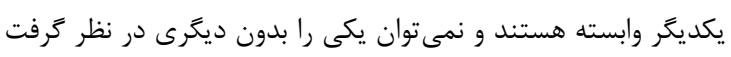

يُروهش هاى اندكى درباره اثربخشى برنامه توانبخشى دوسا-هو انجام

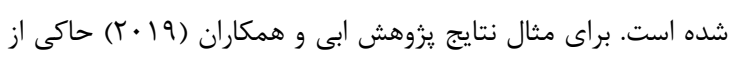

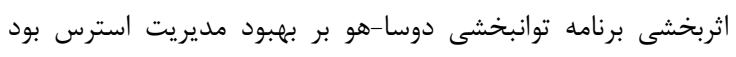

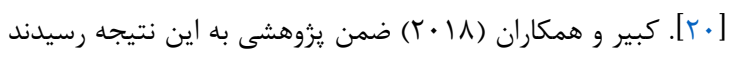

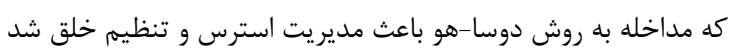

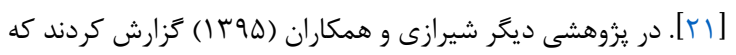

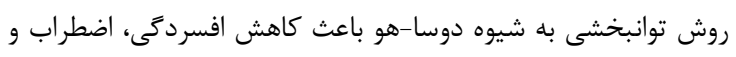

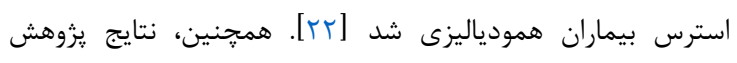

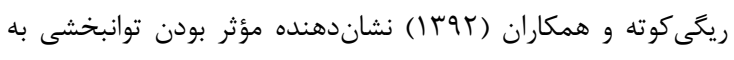

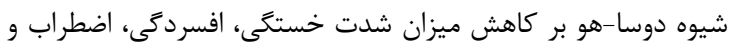

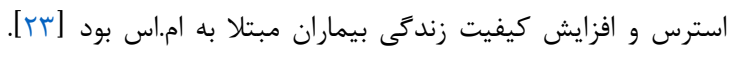

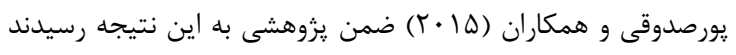

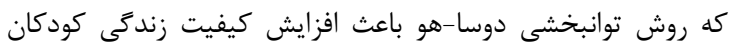

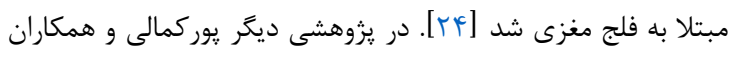

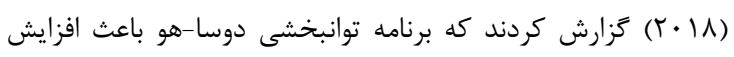

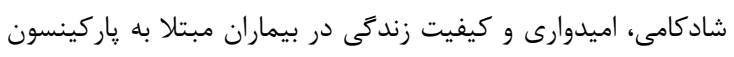

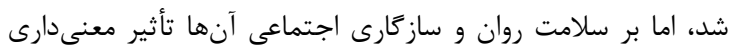

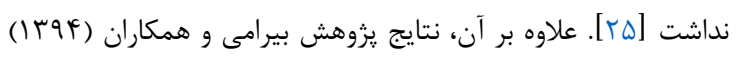

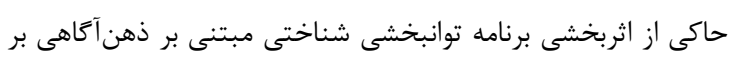

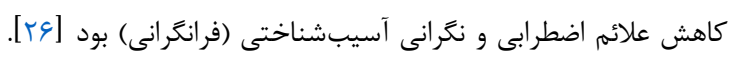

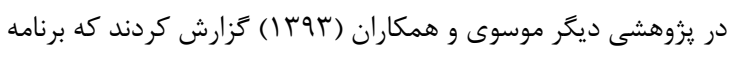

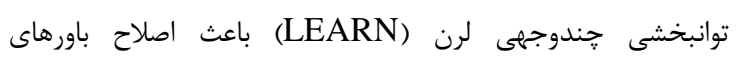

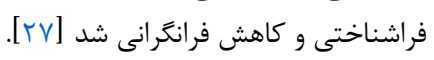

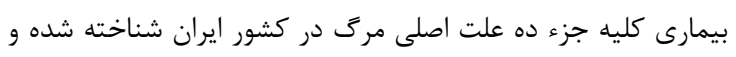

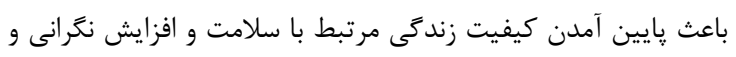

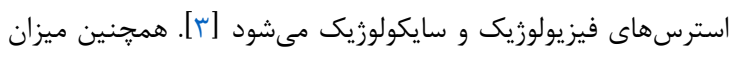

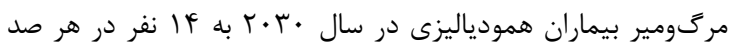

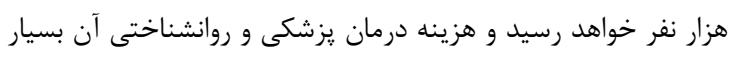

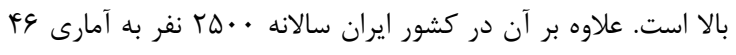

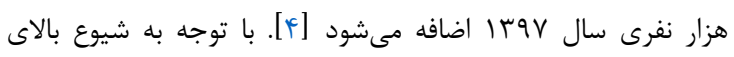

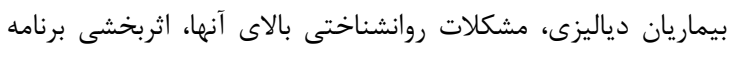

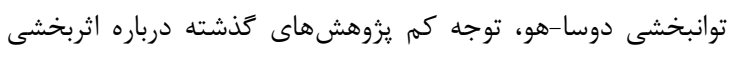

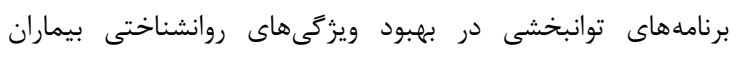

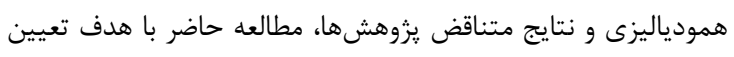

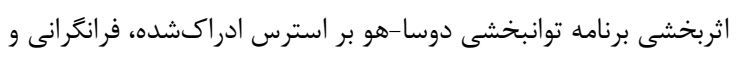
كيفيت زندكى بيماران همودياليزى انجام شد.
جايكزينى كليه مى توان به دياليز و پييوند كليه اشاره كرد كه همودياليز

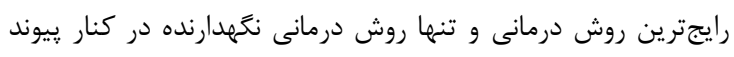

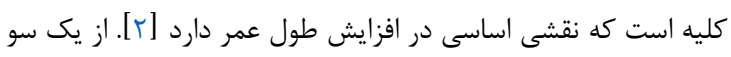

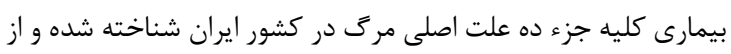

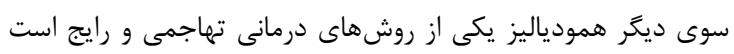

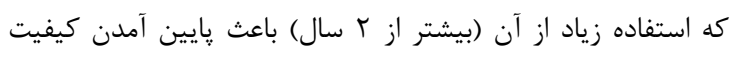

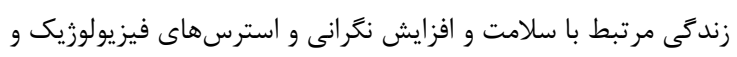

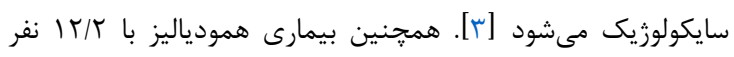

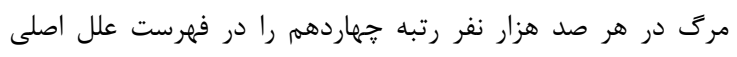

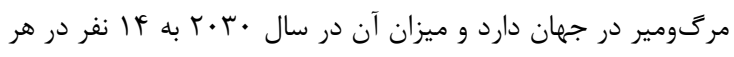

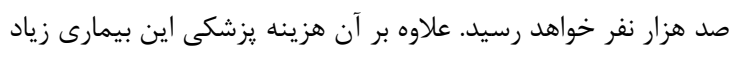

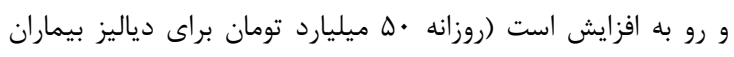

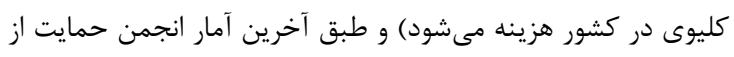

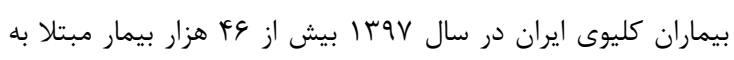

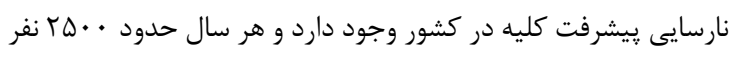

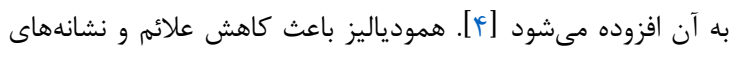

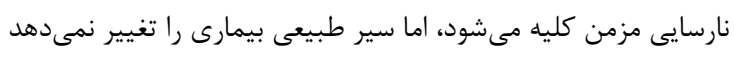

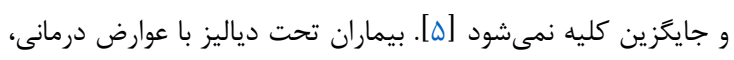

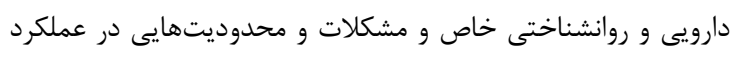

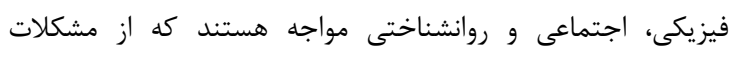

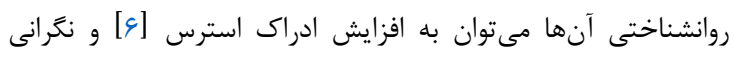

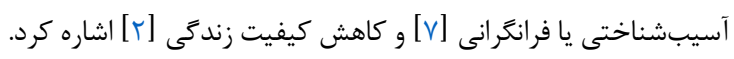

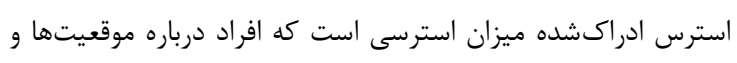

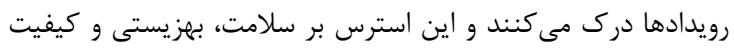

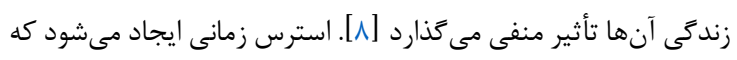

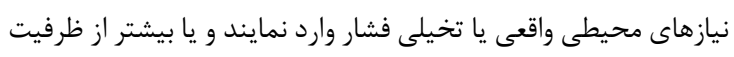

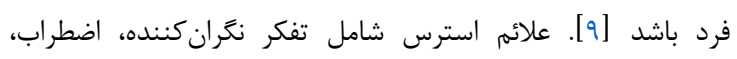

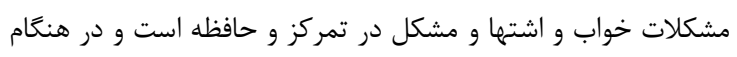

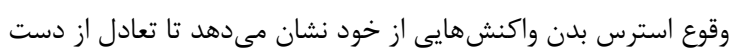

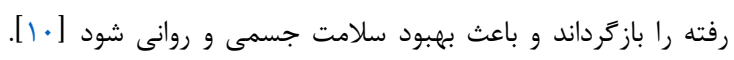

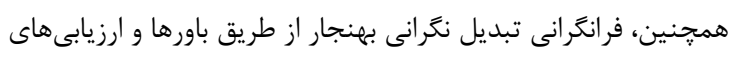

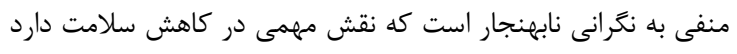

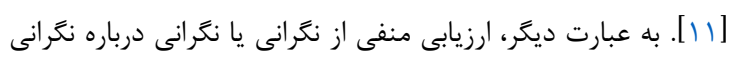

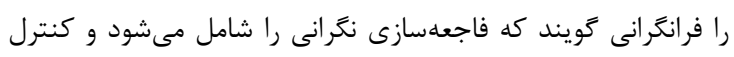

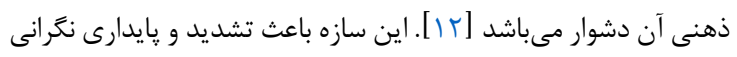

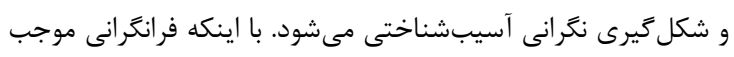

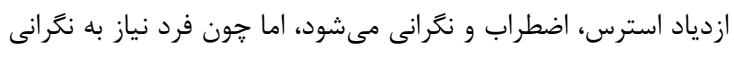

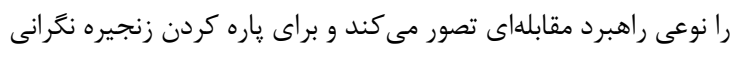

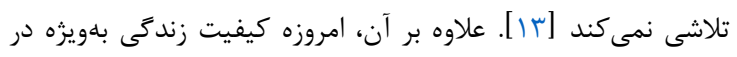

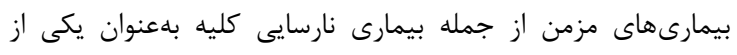

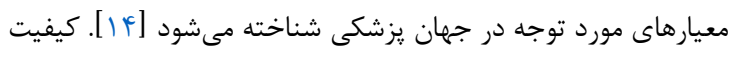

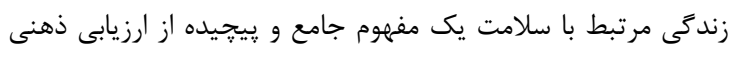

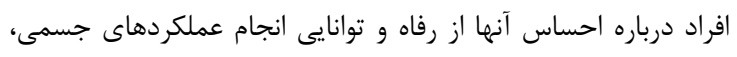

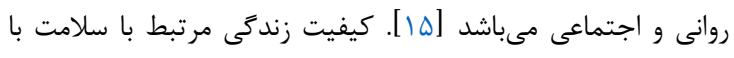

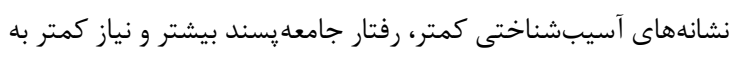
فعاليتهاى تاييدطلبانه توسط ديكران ارتباط دارد [19]. 
كيفيت زندگى مرتبط با سلامت پاسخ دهند و تعداد ع ب نفر از آنها كه

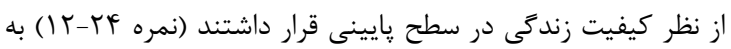

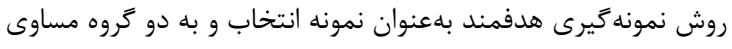

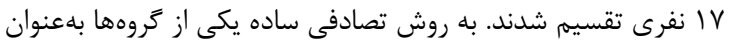

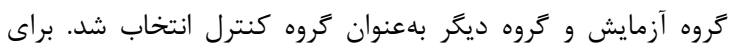

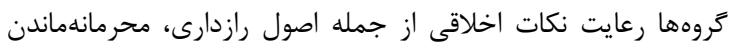

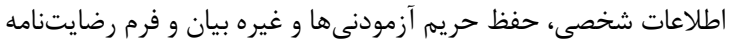

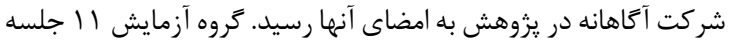

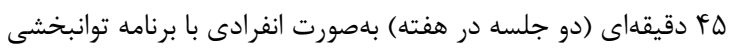
دوسا-هو توسط يك متخصص روانشناسى بالينى داراى مدرى بـ برنامه

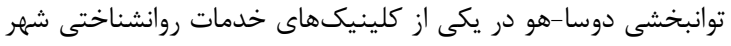

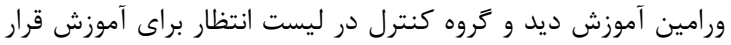

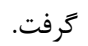
در هر جلسه لها دقيقه ابتدايى به مشاوره در مورد نحوه برقرارى رابطه

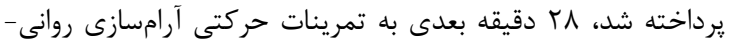

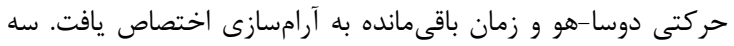

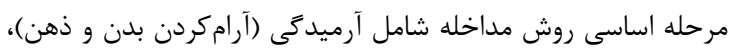

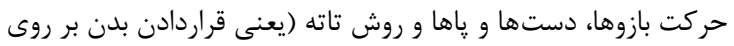

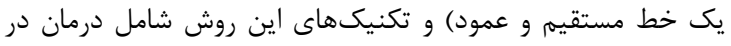

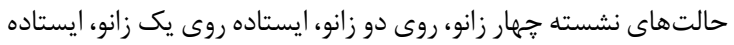

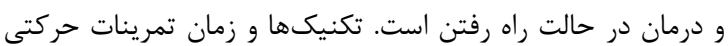

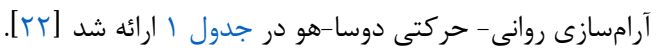

\section{روش كار}

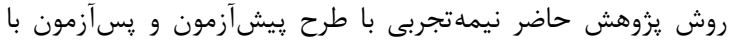

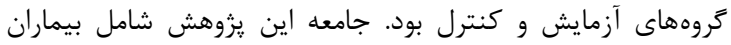

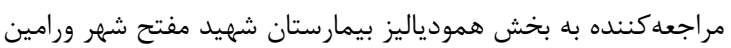

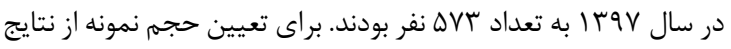

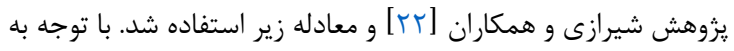

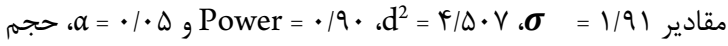

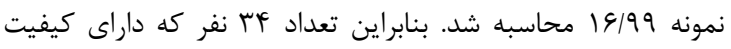

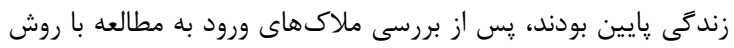

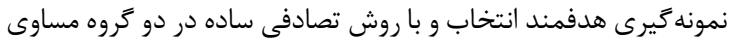

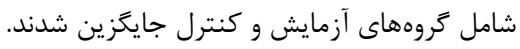
$\mathrm{n}=\frac{2 \sigma^{2}\left(z_{1-\frac{\alpha}{2}+} z_{1-\beta}\right)^{2}}{d^{2}}$

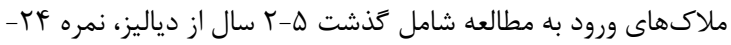
r ا در يرسشنامه كيفيت زندگى مرتبط با سلامت، حداقل تحصيلات

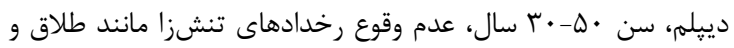

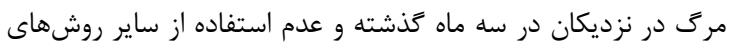
درمانى بلهور همزمان و ملاكهاى خروج از مطالعه شامل انصراف از

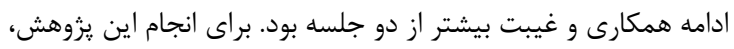

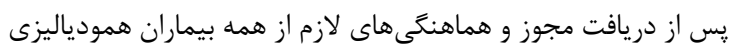

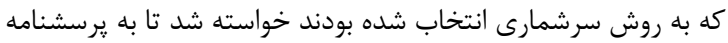

جدول ا. تكنيكهاى اجراشده مداخله دوسا-هو براى بيماران همودياليزى

مدت زمان جلسات و تكنيكها

\section{دقيقه V}

دقيقه د د د V V

دقيقه V دقيقه V

V دقيقه V

V دقيقه V

V دقيقه

V دقيقه V

V دقيقه V V V

دقيقه V

V دقيقه

V دقيقه

V دقيقه V

دقيقه V

V دقيقه

V دقيقه

V دقيقه V

جلسات ب-1

ا. بالا و پايين آوردن دست در حالت درازكش (Oda-age) Y. Tالا و پايين آوردن شانهها (Kata-age) r. جلو و بالاو باينب بردن شانهها و بالا تنه در حالت درازكش (Kukan-no-hineri)

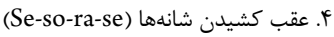

جلسات f-9

1. جلو و عقب بردن شانهها و بالا تنه در حالت درازكش (Kukan-no-hineri)

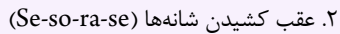
r. بالا و קايين آوردن شانهها (Kata-age)

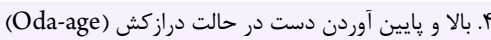

ج-9 جلسات

1. جلو و عقب بردن شانهها و بالا تنه در حالت درازكش (Kukan-no-hineri) r. ايستادن روى دو زانو و حركتدادن باسن (Hizatachi)

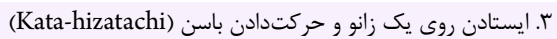
f أ. باز و بسته كردن قفسه سينه و كتف (Mune-hiraki) جلسات 11-1.1

I. كشيدن بالا تنه به طرف جلو و طرفين در حالت نشسته (Zai) r. ايستادن روى دو زانو و حركتدادن باسن (Hizatachi)

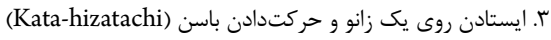

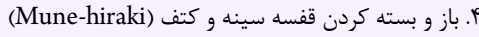

يرسشنامه استرس ادراكشده: اين ابزار توسط كوهن و همكاران

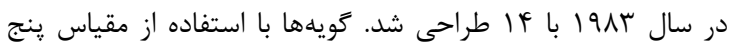
گزينهاى ليكرت از صفر تا جههار نمرهگذارى مىشوند و نمره ابزار با مجموع نمره گويهها محاسبه (دامنه نمرات و
براى جمعآورى دادهها از فرم مشخصات جمعيتشناختى شامل

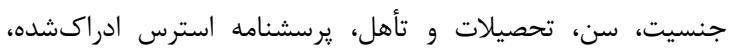

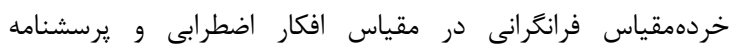
تجديدنظرشده كيفيت زندگى مرتبط با سلامت استفاده شد. 


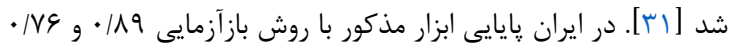

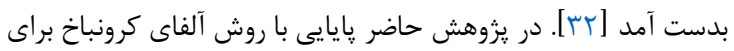

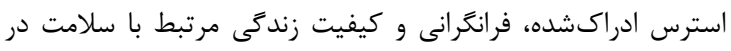

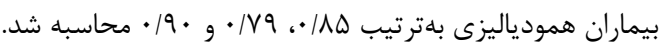

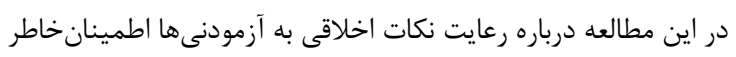

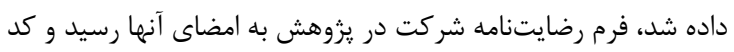

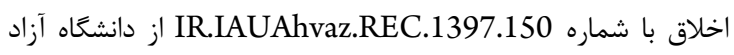

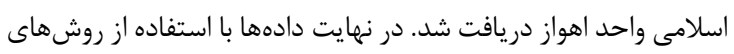

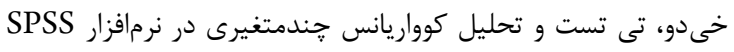

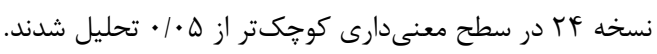

\section{يافتهها}

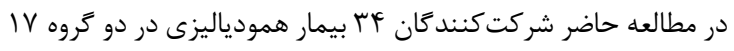

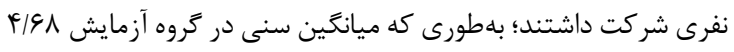

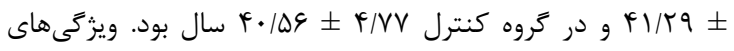
جمعيتشناختى جنسيت، سن، تحصيلات و تأهل واحدهاى يزوهش ورن در جدول r ارائه شد. طبق نتايج جدول r، گروههاى آزمايش و كنترل از نظر جنسيت، سن،

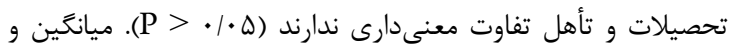

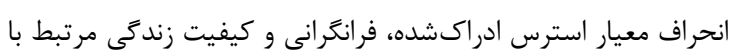
سلامت واحدهاى يزوهش در جدول بار ارائه شد.
معناى استرس ادراكشده بيشتر است. روايى همكراى ابزار با بأبأ

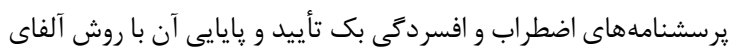

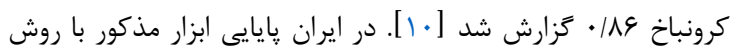

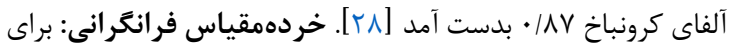

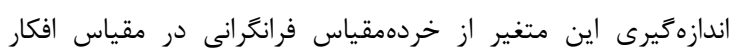

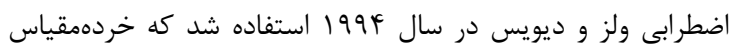

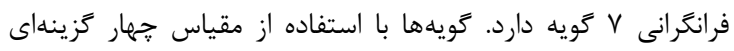

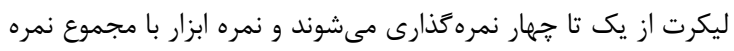

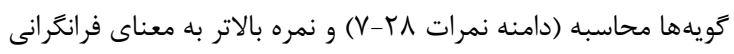

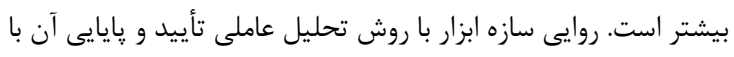

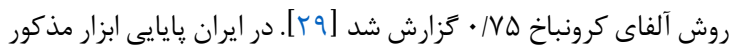

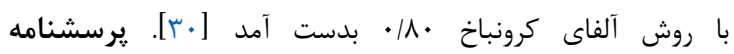

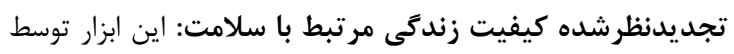

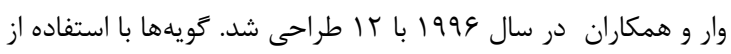

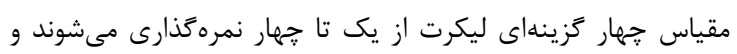

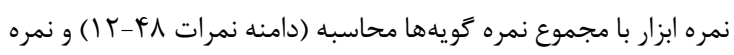

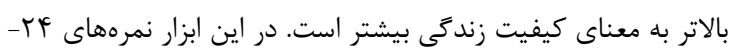

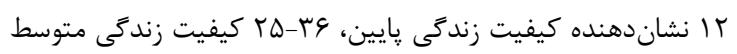

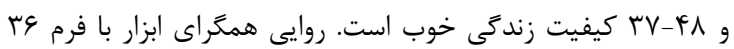

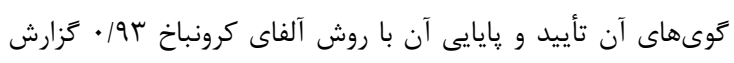

\begin{tabular}{|c|c|c|c|c|}
\hline & & & & جنسيت \\
\hline \multirow[t]{3}{*}{$P>\cdot / \cdot \Delta$} & $(\Delta N / A T))$. & $(\Delta T / Q \Psi) q$ & مرد & \\
\hline & $(\mid q \backslash / \backslash \Lambda) \vee$ & $(F V / \cdot G) \wedge$ & زن & \\
\hline & & & & سن (سال) \\
\hline \multirow[t]{5}{*}{$\mathrm{P}>\cdot 1 \cdot \Delta$} & $(T / / \Delta T)^{F}$ & $(T / \Delta T)^{f}$ & rו-ra & \\
\hline & $(T / / \Delta T)^{F}$ & $(I V / V \Delta) r$ & $r q-\varphi_{\text {. }}$ & \\
\hline & $(r q / F) Q \Delta$ & $(r \Delta / r q) \varphi$ & PI-Fa & \\
\hline & $(T / \Delta T)^{F}$ & $(T / \Delta T)^{\varphi}$ & $\varphi q-\alpha$. & \\
\hline & & & & تحصيلات \\
\hline \multirow[t]{4}{*}{$\mathrm{P}>\cdot / \cdot \Delta$} & $(\& \mid / / \Lambda) V$ & $(\& V / \cdot 9) \wedge$ & دييلم & \\
\hline & $(T / \Delta T)^{F}$ & $(r q / q)) \Delta$ & فوقدييلم & \\
\hline & $(r \Delta / r q) \varphi$ & $(T r / \Delta T)^{q}$ & كارشناسى & \\
\hline & & & & تأهل \\
\hline \multirow[t]{2}{*}{$\mathrm{P}>\cdot / \cdot \mathrm{\Delta}$} & $(\Delta N / A T))$. & $(V \cdot / \Delta q) I r$ & متأهل & \\
\hline & $(\& 1 / / \Lambda) V$ & $((q / / q)) \Delta$ & مجرد & \\
\hline
\end{tabular}

\begin{tabular}{|c|c|c|c|}
\hline معنىدارى T-Test & " كروه كنترل " & " كروه آزمون" & متغيرها \\
\hline & & & استرس ادراكشده \\
\hline$P>\cdot / \cdot \Delta$ & $r \mu / / \Delta \pm F / \Psi_{1} \Lambda$ & $r F / V G \pm F / T q$ & بيش آزمون \\
\hline \multirow[t]{2}{*}{$\mathrm{P}<\cdot \cdot \cdot \cdot 1$} & $r \varphi / \cdot 1 \pm r / r r$ & $I V / r \Delta \pm T / A T$ & 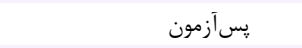 \\
\hline & & & فرانكَرانى \\
\hline $\mathrm{P}>\cdot / \cdot \Delta$ & $\mid \varepsilon / \Delta \varphi \pm r / \Delta \gamma$ & $19 / 9 \gamma \pm r / V$. & 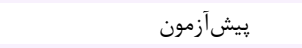 \\
\hline \multirow[t]{2}{*}{$\mathrm{P}<\cdot / . .1$} & $\mid 9 / V T \pm r / 99$ & $|1 / F| \pm r / r q$ & يس آزمون \\
\hline & & & كيفيت زندگى مرتبط با سلامت \\
\hline$P>\cdot / \cdot \Delta$ & $|9 / \Delta| \pm r / \Delta V$ & $r \cdot / 49 \pm 1 / 9 \Delta$ & ي بيش آزمون \\
\hline $\mathrm{P}<\cdot / \cdot \cdot 1$ & $\mid N / V F \pm 1 / M \Lambda$ & $r I / r F \pm r / V G$ & يس آزمون \\
\hline
\end{tabular}


برابرى واريانسها بر اساس آزمون لوين تأييد شدند (ه • P > P).

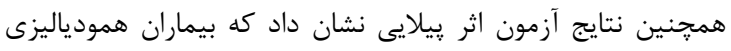

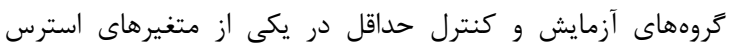

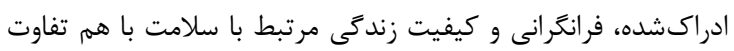

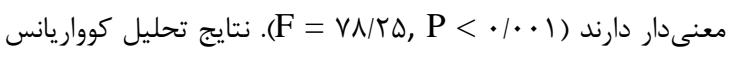

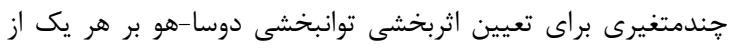

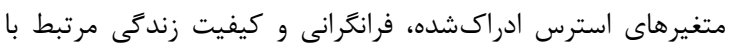
سلامت در واحدهاى يزوهش در جدول ؟ أ ارائه شد.
طبق نتايج جدول ب، گروههاى آزمايش و كنترل در مرحله پِيش آزمون

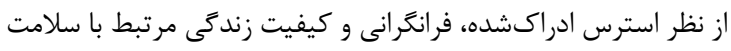

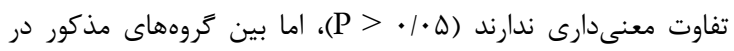

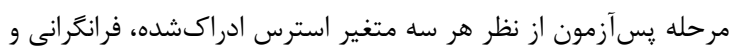

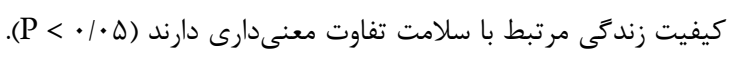

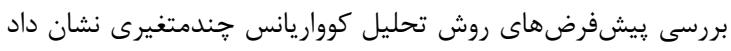

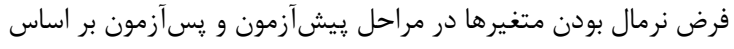

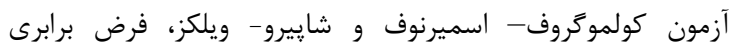

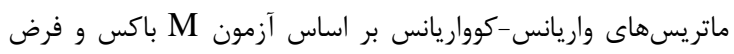

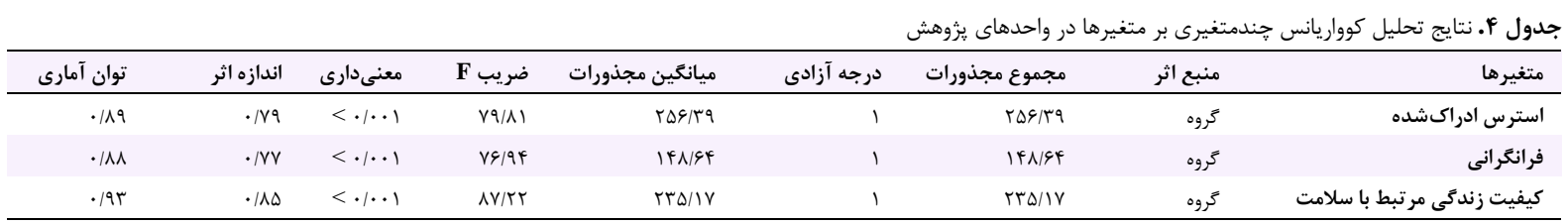

همجنين، نتايج مطالعه حاضر نشان داد كه برنامه توانبخشى دوسا-هو

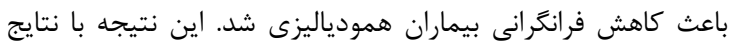

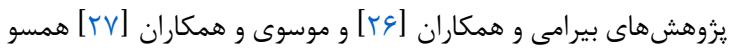

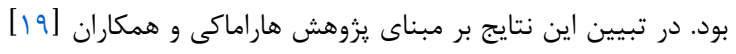

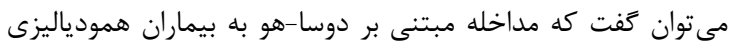

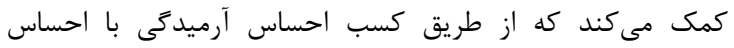

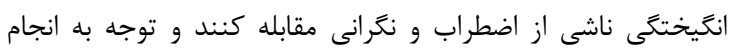

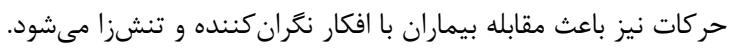

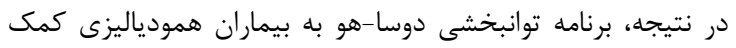

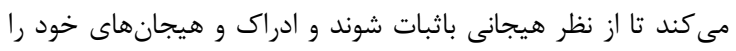

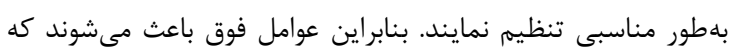

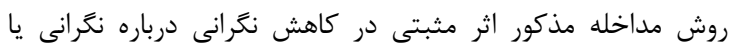

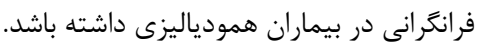

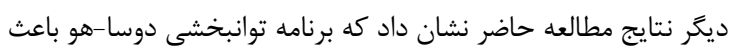

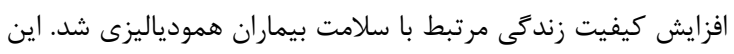

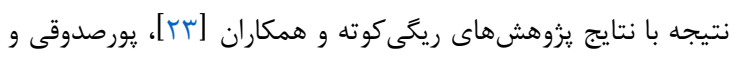

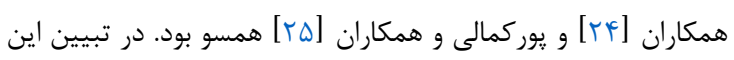

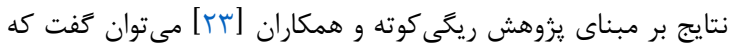

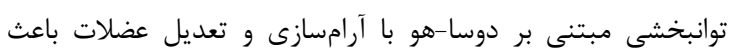

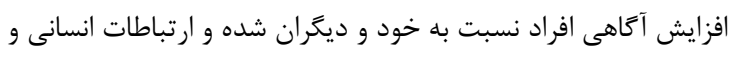

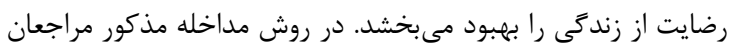

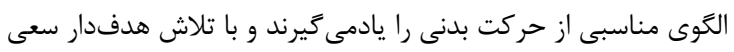

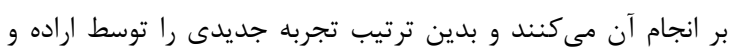

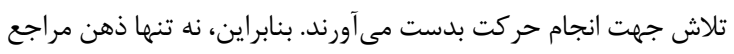

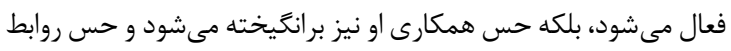

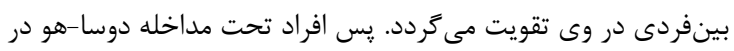

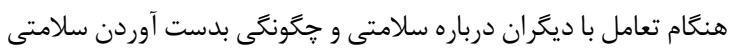

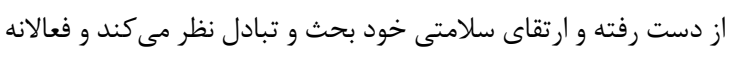

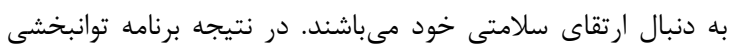

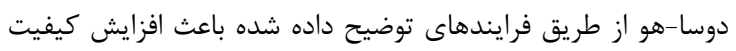
زندكى مرتبط با سلامت بيماران همودياليزى مى فى فرود.
طبق نتايج جدول ع، گروههاى آزمايش و كنترل از نظر هر سه متغير

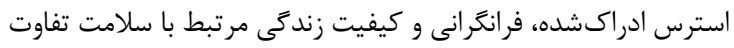

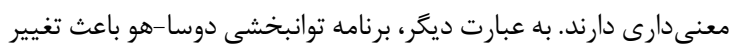

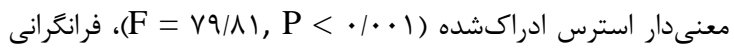

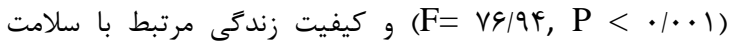

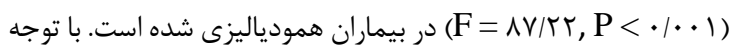

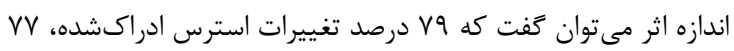

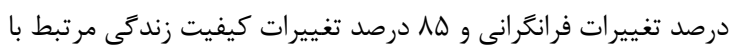

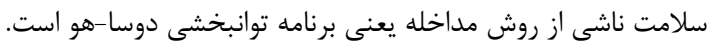

با توجه به آمار بالاو ورو به افزايش بيماران كليه و هزينه بالاى دياليز براى بيماران كليوى، يزوهش حاضر با هدف تعيين اثربخشى برنامه باريه

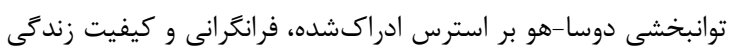

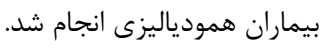
نتايج مطالعه حاضر نشان داد كه برنامه توانبخشى دوسام دادي-هو باعث

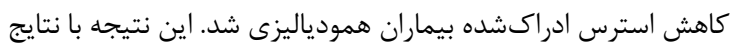

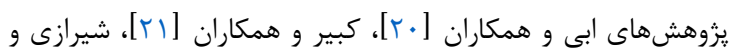

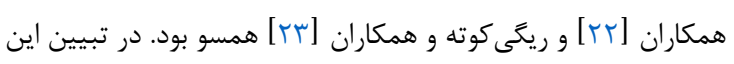

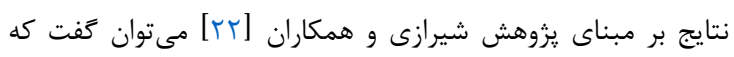

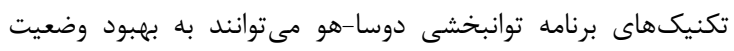

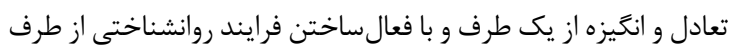

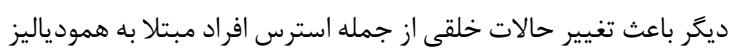

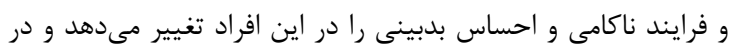

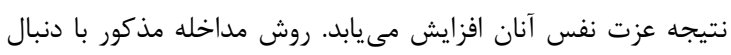

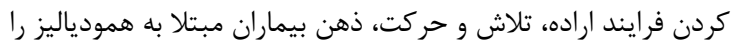

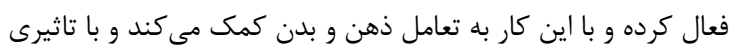

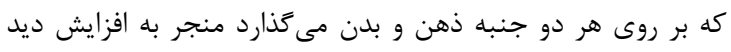

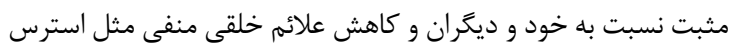
در بيماران همودياليزى مىشود. 


\section{حمايت مالى \\ اين يروهش با هزينه نويسندكان انجام شده است. \\ سهم نويسندكان}

خانمها آزيتا بهجت و شهلا كربلايى صالح: هماهنكى براى انجام

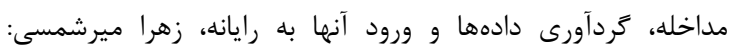

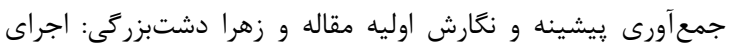
مداخله، تحليل دادها، نحارش نهايى مقاله، سابميت، بِيخيرى و اصلاح

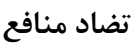

با توجه به اظهارنظر نويسندكان بين آنها تضاد منافعى وجود ندارد. كاربرد عملى مطالعه

با توجه به شيوع بالاو ورزافزون بيمارى كليه و مشكلات روانشناختى

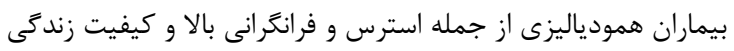

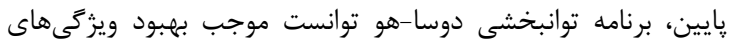

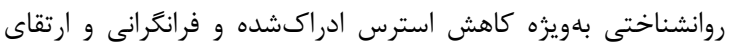

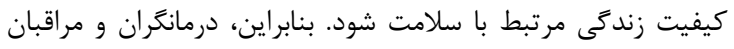

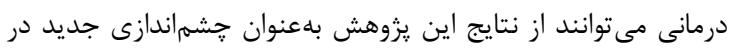
جهت ارتقاى شاخصهاى سلامت و حتى بيشگيرى از آنهان آنها در بيماران

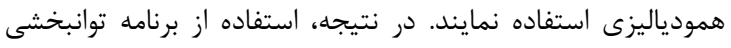

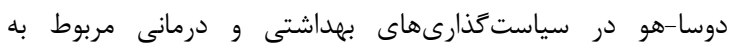

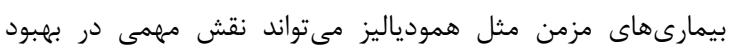

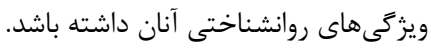
تشكر و قدردانى نويسندكان بدين وسيله تشكر و قدردانى خود را از شركت كنندكان در

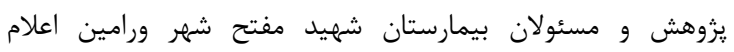
مىنمايند.

\section{References}

1. Shimizu A, Sonoda S, Muraoka Y, Setoyama K, Inoue K, Miura $\mathrm{T}$, et al. Bleeding and ischemic events during dual antiplatelet therapy after second-generation drug-eluting stent implantation in hemodialysis patients. J Cardiol. 2019;73(6):470-8. doi: 10.1016/j.jjcc.2018.12.009 pmid: 30591321

2. Cepeda Marte JL, Javier A, Ruiz-Matuk C, PaulinoRamirez R. Quality of Life and Nutritional Status in diabetic patients on hemodialysis. Diabetes Metab Syndr. 2019;13(1):576-80. doi: 10.1016/j.dsx.2018.11.020 pmid: 30641769

3. Mousavi Bazaz SM, Keyvanlo Z, Keykhosravi A, Neamatshahi M, Asadi A, Neamatshahi M. Assessment quality of life heamodialysis patients and and Influencing Factors in Mashhad University of Medical Sciences hospitals in 2017. [Persian]. J Sabzevar Univ Med Sci. 2019;26(4):413-20.
از محدوديتهاى مهمم مىتوان به روش نمونهَيرى هدفمند، حجم

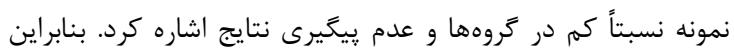

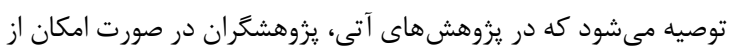

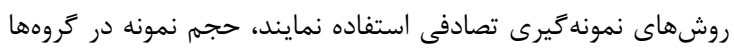

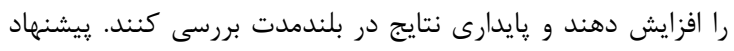

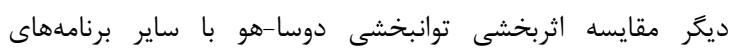

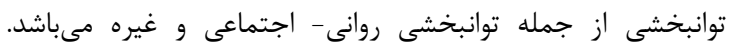

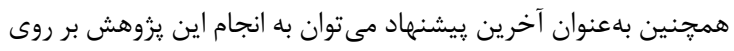

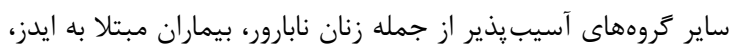

$$
\text { ديابت و غيره اشاره كرد. }
$$

\section{نتيجه كيرى}

برنامه توانبخشى دوسا-هو باعث كاهش استرس ادراكشده و فرانكرانى

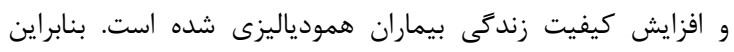

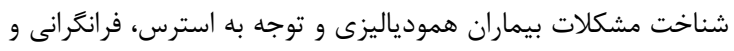

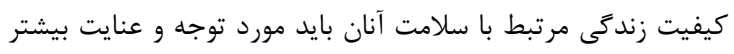

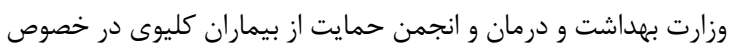

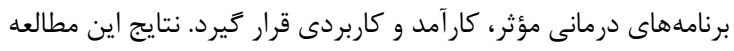

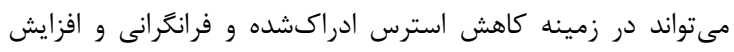
كيفيت زندگى مرتبط با سلامت كمك دمننده زماند باشد. بنابراين، توصيه

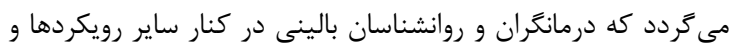

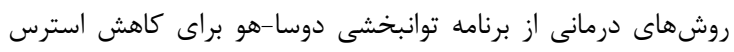

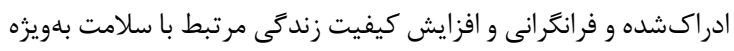

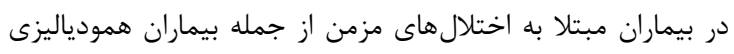

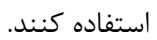

ملاحظات اخلاقى

در اين مطالعه، نكات اخلاقى طبق : بروتكلهاى اخلاقى مطالعات

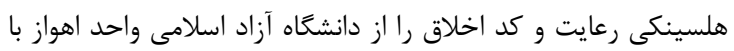

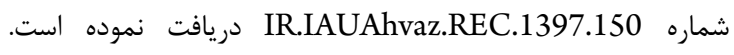
همجنين، فرم رضايتنامه شركت در يزوهش توسط همه آزمودنىها

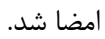

4. Soodmand M, Ghasemzadeh G, Mirzaee S, Mohammadi M, Amoozadeh Lichaei N, Monfared A. Self-care Agency and Its Influential Factors in Hemodialysis Patient. [Persian]. Iran J Nurs. 2019;32(118):86-95. doi: 10.29252/ijn.32.118.86

5. Wong MMY, Thijssen S, Wang Y, Usvyat LA, Xiao Q, Kotanko P, et al. Prediction of Mortality and Hospitalization Risk Using Nutritional Indicators and Their Changes Over Time in a Large Prevalent Hemodialysis Cohort. J Ren Nutr. 2020;30(1):69-78. doi: 10.1053/j.jrn.2019.01.013 pmid: 30852118

6. Musa AS, Pevalin DJ, Al Khalaileh MAA. Spiritual WellBeing, Depression, and Stress Among Hemodialysis Patients in Jordan.J Holist Nurs. 2018;36(4):354-65. doi: 10.1177/0898010117736686 pmid: 29173010

7. Weng $\mathrm{CH}$, Lu KY, Hu CC, Huang WH, Wang IK, Yen $\mathrm{TH}$. Bone marrow pathology predicts mortality in chronic 
hemodialysis patients. Biomed Res Int. 2015;2015:160382. doi: 10.1155/2015/160382 pmid: 25802835

8. Catabay CJ, Stockman JK, Campbell JC, Tsuyuki K. Perceived stress and mental health: The mediating roles of social support and resilience among black women exposed to sexual violence. J Affect Disord. 2019;259:143-9. doi: 10.1016/j.jad.2019.08.037 pmid: 31445340

9. Lonnberg G, Jonas W, Unternaehrer E, Branstrom R, Nissen E, Niemi M. Effects of a mindfulness based childbirth and parenting program on pregnant women's perceived stress and risk of perinatal depression-Results from a randomized controlled trial. J Affect Disord. 2020;262:133-42. doi: 10.1016/j.jad.2019.10.048 pmid: 31733457

10. Lovell B, Wetherell MA. Affiliate stigma, perceived social support and perceived stress in caregivers of children with autism spectrum disorder: A multiple mediation study. Arch Psychiatr Nurs. 2019;33(5):31-5. doi: 10.1016/j.apnu.2019.08.012 pmid: 31711591

11. Spada MM, Challoner H, Nikcevic A, Fernie BA. Metacognitive beliefs about worry and pain catastrophising as mediators between neuroticism and pain behaviour. Clin Psychol. 2016;20(3):138-46. doi: 10.1111/cp.12081

12. Myhr P, Hursti T, Emanuelsson K, Lofgren E, Hjemdal O. Can the Attention Training Technique Reduce Stress in Students? A Controlled Study of Stress Appraisals and Meta-Worry. Front Psychol. 2019;10:1532. doi: 10.3389/fpsyg.2019.01532 pmid: 31354569

13. Esbjorn BH, Lonfeldt NN, Nielsen SK, Reinholdt-Dunne ML, Somhovd MJ, Cartwright-Hatton S. Meta-worry, worry, and anxiety in children and adolescents: relationships and interactions. J Clin Child Adolesc Psychol. 2015;44(1):145-56. doi: 10.1080/15374416.2013.873980 pmid: 24555865

14. Namdar A, Beigizadeh S, Najafipour S. Health- related quality of life in dialysis patients. [Persian]. JMJ. 2012;10(4):19-27. doi: 10.29252/jmj.10.4.19

15. Hamby S, Taylor E, Mitchell K, Jones L, Newlin C. Health-Related Quality of Life among Adolescents as a Function of Victimization, other Adversities, and Strengths. J Pediatr Nurs. 2020;50:46-53. doi: 10.1016/j.pedn.2019.11.001 pmid: 31756596

16. Messina S, Frongia AL, Antonaci L, Pera MC, Coratti G, Pane $M$, et al. A critical review of patient and parent caregiver oriented tools to assess health-related quality of life, activity of daily living and caregiver burden in spinal muscular atrophy. Neuromuscul Disord. 2019;29(12):940-50. doi: 10.1016/j.nmd.2019.10.001 pmid: 31791871

17. Fujino H. Psychological Support for Young Adults with Down Syndrome: Dohsa-Hou Program for Maladaptive Behaviors and Internalizing Problems. Front Psychol. 2017;8:1504. doi: 10.3389/fpsyg.2017.01504 pmid: 28919875

18. Bokhan NA, Voevodin IV, Aslanbekova NV. [The dynamics of addictions in students and possibilities of reflecting it in psychorehabilitative programs]. Zh Nevrol
Psikhiatr Im S S Korsakova. 2014;114(5 Pt 2):29-32. pmid: 24988972

19. Haramaki Y, Kabir RS, Abe K, Yoshitake T. Promoting Self-Regulatory Management of Chronic Pain Through Dohsa-hou: Single-Case Series of Low-Functioning Hemodialysis Patients. Front Psychol. 2019;10:1394. doi: 10.3389/fpsyg.2019.01394 pmid: 31281283

20. Abe K, Kabir RS, Haramaki Y. Referencing the body for mood state regulation: an examination of stress management using Dohsa-hou as a primary prevention program for nurses. Environ Occupation Health Pract. 2019;1(2):55-60. doi: 10.1539/eohp.2019-0010-GP

21. Kabir RS, Haramaki Y, Ki H, Ohno H. Self-Active Relaxation Therapy (SART) and Self-Regulation: A Comprehensive Review and Comparison of the Japanese Body Movement Approach. Front Hum Neurosci. 2018;12:21. doi: 10.3389/fnhum.2018.00021 pmid: 29472851

22. Shirazi M, Koohkan Azim H, Khosravani E. Effectiveness of psychological rehabilitation, using Dohsa-Hou, on hemodialysis patients' depression, anxiety, and stress in Zahdan city. [Persian]. J Birjand Univ Med Sci. 2016;23(2):130-40.

23. Rigikouteh B, Yazdkhasti F, Etemadifar M. The effectiveness of Dohsa-hou psychological rehabilitation program on severity of fatigue, depression, anxiety, stress and improve the quality of life in subjects with Multiple Sclerosis (MS). [Persian]. J Res Rehabil Sci. 2013;9(3):445-58.

24. Poursadoughi A, Dadkhah A, Pourmohamadreza-Tajrishi M, Biglarian A. Psycho-rehabilitation method (Dohsahou) and quality of life in children with cerebral palsy. Iran Rehabil J. 2015;13(2):28-33.

25. Pour Kamali T, Yazdkhasti F, Oreyzi HR. A comparison of effectiveness of Dohsa-hou and the alexander technique on happiness, social adjustment, hope, mental health, and quality of life in patients with parkinson's disease. Japan Psychol Res. 2018;60(2):87-98. doi: 10.1111/jpr.12184

26. Bayrami M, Movahedi Y, Kazimi Razai S, Esmaili S. The effect of mindfulness cognitive therapy on pathological worry and anxiety symptoms in students with generalized anxiety disorder. [Persian]. IJRN. 2015;2(1).

27. Mousavi E, Alipour A, Zare H, Agah Heris M, Janbozorgi M. Effectiveness Of LEARN multifaceted intervention in modifying meta-cognition and the meta-worry beliefs. [Persian]. Advance Cognitive Sci. 2014;16(1):39-48.

28. Halajani F, Jameinezhad F, DashtBozorgi Z, Peymani Foroshani R, EsmaeiliShad B. The effect of cognitive rehabilitation group training on perceived stress, depression and quality of life among primigravid women with pregnancy anxiety. [Persian]. Iran J Rehabil Nurs Sci. 2019;6(1):10-7.

29. Wells A, Davies MI. The thought control questionnaire: A measure of individual differences in the control of unwanted thoughts. Behav Res Ther. 1994;32:871-8. doi: 10.1016/0005-7967(94)90168-6 pmid: 7993332

30. Shirali Sh, DashtBozorgi Z. Effect of existential group therapy on distress tolerance, existential anxi-ety, meta- 
worry and health worry in elderly women with cardiovascular disease. [Persian]. Cardiovascular Nursing Journal. 2019;7(4):24-33.

31. Ware J, Jr., Kosinski M, Keller SD. A 12-Item Short-Form Health Survey: construction of scales and preliminary tests of reliability and validity. Med Care.
1996;34(3):220-33. doi: 10.1097/00005650199603000-00003 pmid: 8628042

32. Rezaee M, Arabi S, Sahaf R, Rassafiani M, Hosseini H, Mirzakhany N, et al. Validity and reliability of the Persian version of measurement of the quality of life of people with disabilities. [Persian]. Pejouhandeh. 2014;19(2):91-8. 